\title{
Female cancers. A perspective from the Latin South
}

\author{
Yolanda Eraso (*) and Luiz Antonio Teixeira (**) \\ $\left(^{*}\right)$ Faculty of Health and Life Sciences, Oxford Brookes University. yolandaeraso@brookes.ac.uk \\ ${ }^{* *}$ ) Casa de Oswaldo Cruz / Fiocruz. Rio de Janeiro. teixeira@fiocruz.br
}

Dynamis

[0211-9536] 2014; 34 (1): 17-24

http://dx.doi.org/10.4321/S0211-95362014000100002

In the last decade or so, the history of cancer has emerged as a distinctive topic of study that has rejuvenated the field of the "history of diseases» thanks to the work of a heterogeneous group of medical and social historians, sociologists, anthropologists and health professionals primarily based in Europe and Anglo-Saxon countries. Such a multi-disciplinary approach has returned a kaleidoscope of perspectives where cancer and its history seem to harbour some meaningful insights into the many unanswered questions that the disease poses at the present. In so doing, these surveys are contributing to expand the analysis of the history of diseases by drawing attention to chronic and non-communicable diseases whose relevance have been somewhat neglected in comparison to the attention given to contagious and infectious diseases.

A good deal of the recent scholarship has concentrated particularly in the history of female cancers, most specifically, gynaecological cancers (breast, cervix, ovarian and uterus) and thus has begun to outline and chart the trajectories of those particular cancers on wider contemporary notions of women's health, and as an example of the impact of cancer in societies more broadly ${ }^{1}$. There is, however, some reason for the growing literature

1. For the history of female cancers only there is a considerable corpus of literature in journal articles, papers, and PhD dissertations. An incomplete enumeration of monographs since the year 2000, includes: Lerner, Barron. The breast cancer wars: Hope, fear, and the pursuit of a cure in twentieth-century America. New York: Oxford University Press; 2001; Olson, James. Bathsheba's breast: Women, cancer, and history. Baltimore: Johns Hopkins University 
examining female cancers as privileged sites of cancer history: early on in the twentieth century, gynaecological cancers became often associated with the disease and, in the following decades, observations about their identification, diagnosis, treatment, and prognosis underpinned, to a great extent, general notions about cancer as a disease more broadly. For example, the «local to general» theory that explained the progression of cancer as a disease was a principle grounded in the Halstedian concept of breast cancer biology, whilst the efficacy of screening in asymptomatic populations was first introduced through cervical and breast cancer campaigns. Arguably, the relative accessibility of tumours of the breast and the cervix to doctors' observation alongside the demographic, biological and racial concerns with women's maternal function, and a long tradition of medicalisation of the female sex more broadly have helped framed the association of cancer as a women's disease. Yet the latter does not mean that a gender component has dominated the interpretations of the disease in a similar way to that observed in other gendered associations, notably, women and mental health disorders where hysteria became quintessentially identified as a female malady. Historical examinations of cancer have been cautious in portraying the gender components of the disease as permeating all the initiatives in «the fight against cancer». And here both the national and local reconstructions of the disease have prevented from the formulation of sweeping generalisations.

More recently, historians of cancer in Latin America are contributing too to the historical accounts of the disease, documenting some of its distinctive features in a region that could be regarded as an exemplary instance of transnationalism. In this sense, Latin America offers a fruitful locus to explore issues of spatiality in medical knowledge that go beyond a mere recount of the commonality and the differences observed in the control of the disease in relation to the European and the American context

Press; 2005; Gardner, Kirsten. Early detection: Women, cancer, and awareness campaigns in the twentieth-century United States. Chapel Hill: University of North Carolina Press; 2006; Aronowitz, Robert. Unnatural history: Breast cancer and American society. Cambridge: Cambridge University Press; 2007; Reynolds L.A.; Tansey, E.M., eds. History of cervical cancer and the role of the human papillomavirus, 1960-2000 [Vol. 38 of the Witness Seminar series]. London: Wellcome Trust; 2009; Bryder, Linda. Women's bodies and medical science: An inquiry into cervical cancer. Basingstoke: Palgrave Macmillan; 2010; Löwy, llana. Preventive strikes. Women, precancer, and prophilactic surgery. Baltimore: John Hopkins University Press; 2010; Löwy, Ilana. A woman's disease: The history of cervical cancer. Oxford: Oxford University Press; 2011. 
that by and large have informed Latin American approaches. Grounded in a long tradition of academic exchanges and medical training abroad, the dissemination of industrialised countries' treatments, technologies, and scientific notions provided Latin American cancerology with conceptual and empirical elements whose local assemblage had implications in- and outside the region's borders. Indeed, like in other areas of medical knowledge and international health ${ }^{2}$, the dynamic way in which exchanges occurred have yielded contributions and returns to European and American contexts which have considerably affected the latter in its own approaches to the disease. In the specific case of female cancers, South American countries in particular have been no exception. On the one hand, Argentina and Brazil offer a unique setting to analyse both medical and health policy interventions in female cancers because both countries have pioneered a combined use of diagnostic tools for cervical cancer early detection: the Pap smear test and colposcopy ${ }^{3}$. On the other, it was in Uruguay where mammography was technically improved, alongside the interpretation of core radiological signs of breast cancer, which paved the way for the clinical acceptance (diagnosis) of the technique and later as a screening method, something that was wholeheartedly pursued by the American Cancer Society in the $1970 \mathrm{~s}^{4}$.

The initiatives in Latin American nations to control female cancers thus allow us to observe how local cultures of medicine, health policies and the particularities of the social context differently shaped the utilisation of

2. See amongst others Birn, Anne-Emanuelle; Hochman, Gilberto. Communication, control, and co-operation: (Latin) American interchanges in the history of international health. Canadian Bulletin of Medical History. 2008; 25 (1): 7-16; Kreimer, Pablo. Understanding scientific research on the periphery: towards a new sociological approach? EASST Review. 1998; 17 (4); Rodriguez, Julia. South Atlantic crossings: Fingerprints, science, and the state in turn-of-thecentury Argentina. American Historical Review. 2004; 109 (2): 387-416; Quevedo, Emilio et al. Knowledge and power: The asymmetry of interests of Colombian and Rockefeller doctors in the construction of the concept of «jungle yellow fever», 1907-1938. Canadian Bulletin of Medical History. 2008; 25 (1): 71-109.

3. Eraso, Yolanda. Migrating techniques, multiplying diagnoses: The contribution of Argentina and Brazil to early «detection policy» in cervical cancer. História, Ciências, Saúde-Manguinhos. 2010; 17 (suppl. 1): 33-51; Teixeira, Luiz; Löwy, Ilana. Imperfect tools for a difficult job: Colposcopy, «colpocytology» and screening for cervical cancer in Brazil. Social Studies of Science. 2011; 41 (4): 585-608.

4. Eraso, Yolanda. Imagin(in)g the breast. Mammography and breast cancer in the context of South-North American exchanges. Paper delivered at the History of Medicine Seminars, Department of History and Philosophy of Science. University of Cambridge, January 2012. 
technologies destined to the disease in different geographical places and at different moments. On the one hand, the different regimes of utilisation of the Pap smear test in the public health system and in the sphere of private medicine constitute a good example of the extent to which medical technologies and therapies are not neutral dispositives, but instruments shaped in the interaction of actors in their everyday practices ${ }^{5}$. On the other, the long-term persistence of colposcopy as the first examination in countries such as Brazil, Argentina, Chile and Uruguay and the more recent strategy of «see and treat» for identifying and managing high grade precancerous lesions - in regions where the capacity of the health system is limited-demonstrate how the different forms of appropriation and utilisation of techniques are, in turn, generating new forms of intervention and different policies to control the disease.

The recent flurry of academic and non-academic monographs about cancer and its history may also induce us to think that the subject has gained a status whose contribution may well satisfy an existing demand for disentangling its past. Yet this observation merits some caution. One precise area where the history of cancer most fruitfully can position its strengths is by engaging in an interdisciplinary dialogue, in the, so far, elusive area of health policy-making, and public health interventions. Although historians have rarely been invited to contribute to the debates that precede the design of health care programmes, and despite the fact that policy makers often resort to past interventions as a rationale for their policies, it is important that both historians and policy makers engage in a more efficient use of history. As Virginia Berridge has perceptively considered, the use of history by policy makers «needs to be rethought from both sides of the boundary», and more specifically, for this interaction to become more prosperous « $[\mathrm{h}]$ istorians need to become better at drawing out general principles from their work and at communicating in policy terms» 6 .

In addition, recent global health policies directed to curve the incidence of female cancer (especially cervical cancer) attempt to use technical solutions without a comprehensive understanding of historically-grounded local service structures, the role of professional cultures and the impact of health

5. Clarke, Adele; Fujimura, Joan. The right tools for the job: At work in twentieth-century life sciences. Princeton: Princeton University Press; 1992.

6. Berridge, Virginia. History matters? History's role in health policy making. Medical History. 2008; 52 (3): 311-326 (325). 
inequalities within populations. Situating these studies in detailed national and local case studies is relevant in that they can provide a more contextspecific analysis and thus inform, support or reassess health interventions formulated at a global level. In a time when developing countries are being encouraged to re-organising their health systems structures in order to be able to confront a predicted increase in the burden of the disease, studies of past developments of cancer organisation, its foundations and its changes, can help understand, amongst other things, what drives change in health and what persistently impedes it. By drawing on a series of case studies, the articles gathered in this collection highlight the relevance of attending to these aspects to a more cogent understanding of current challenges posed by the disease.

In this sense, this issue of Dynamis proposes to add to this new growing literature of cancer studies by adding depth and detail to the study of one the most emblematic of cancers, gynaecological cancers, as well as indicating diversity in similarly configured health systems. This dossier's case studies span the twentieth and twentieth-first century and concentrate on a series of aspects that emerged as a consequence of the policies implemented at different times to control the disease. Articles explore and assess the historical and social intersections of medicine, public health interventions, specialists, and health systems. In analysing the complex organisation of cancer services, the emergence of new actors and their dispute for professional recognition, and the complexities of designing and implementing preventive policies the dossier aims at offering a historical perspective to current debates on cancer screening strategies in middle and low-resource settings.

We begin with Vanessa Lana's article, which discusses the creation and institutionalisation of a specialised centre, the Hospital Aristides Maltez in the state of Bahia, in the Northeast of Brazil, created by the Bahia League Against Cancer in 1952. Towards the 1950s, the Brazilian health policy in cancer care largely relied on the initiatives of private, philanthropic organisations. In this sense, cancer leagues played an important role in obtaining resources for both the construction and maintenance of specialised hospitals. Lana analyses the campaigns organised by the hospital, its initiatives in the area of cancer research and professional training and highlights the hospital's significance in the control of cancer for the state of Bahia. Her study offers insight into how the hospital became instrumental in setting up a network of studies and professional exchanges with the main gynaecology centres of the Southeast region, in particular, with the Institute of Gynaecology of 
the Federal University of Rio de Janeiro, and the Hospital of Gynaecology of the Faculty of Medicine of University of Minas Gerais, in the state of Belo Horizonte. In addition, the article demonstrates the hospital's pioneering large-scale utilisation of the Pap smear test through the organisation of campaigns in the poorest regions (municipalities) of the Bahia state. In this sense, the analysis shows how the use of the test to reach distant places and the knowledge gathered from the needs associated with such interventions - mainly related to the use of the test in larger populations - facilitated the hospital's contribution in the dissemination of cytology as the first diagnostic tool in the control of the disease.

The article of Luiz Teixeira and Letícia Pumar focuses on the specificities that surrounded the emergence of the cytologist or cyto-technician in Brazil and the difficulties experienced in the professionalisation of the discipline. Following historical studies that have emphasised the different patterns observed in the use of the Pap test as a diagnostic tool in different local contexts, the authors demonstrate that the utilisation of this tool in Brazil was the result of agreements, disputes and negotiations amongst specific professional groups, that is, doctors specialised in different areas, pharmacists, biologists, and cytologists. The first part of the article analyses how the emergence of cytologists as a professional group was linked to the development of the first screening campaigns for cervical cancer in the 1970s. During that time, different initiatives competed for the professionalisation of cytology, which were developed within the area of public health with the aim of supporting and assisting the ongoing preventive campaigns. Those initiatives, however, proved insufficient in gathering a consensus on the required knowledge and activities that should be within the professional domain. The study later focuses on the creation of a labour market for cytologists analysing how its organisation was related to the tensions between professional groups for the monopoly in the reading of slides and in the supervision of the readings, and how these tensions were linked, in turn, to different interests in the fields of public health and private medicine. The article demonstrates how the diverse perspectives about the Pap smear test within different medical specialties and their relation with the dynamics of the labour market shaped the trajectory of the cytology profession, whose institutionalisation, training, and regulation is still incomplete today and subject to intense debates.

Turning to the case of Argentina, the article of Yolanda Eraso explores the first public and private initiatives to organise the control of cancer in the 
country, drawing special attention to the ideas and institutions that outlined the main structures in the fight against female cancers. Through the analysis of different actors and institutions (medical specialists, philanthropists, the cancer league, and state authorities) the article contends, firstly, that unlike industrialised countries, cancer diagnosis, rather than treatment, became instrumental in delineating a model of cancer control in Argentina. Secondly, it explores the idea of path-dependence to frame the discussion about the alleged contemporary fragmentation and lack of cohesion in cancer programmes. This perspective allows the author to trace how the foundations of competing cancer initiatives during the first decades of the twentieth century were consolidated through old and new institutions emerging after the development of Argentina's health system in the 1940s. This, Eraso argues, helped create the conditions for persistent forms of disintegrated policies and services whose path-dependence pattern seem difficult to disrupt.

The emergence of national health systems and their main components at critical moments in time has not often intermingled with historical accounts of specific diseases in Latin America, which have tended to privilege the development of public health policies, the provision of services, medical notions, and/or the development of treatments. How the whole enterprise of disease control operates within the broader goals and functions of contemporary health systems structures also merits additional attention. The federal-provincial/state tension that has characterised much of Argentina and Brazil's health policy coupled in the former with the development of a health system that incorporated various independent models (private, public, and health insurance), which, as current observers have pointed, became an obstacle for the implementation of health policies at national level. The case of Brazil, on the contrary, points to a different story. As the article of Marco Porto and Paula Habib demonstrates, the creation of a Unified Health System in 1988 - Sistema Único de Saúde or SUS - granted universal access and allowed for the federal planning of health policies thus becoming an enabling component for the development of a cervical cancer programme across the country. More specifically, the authors focus on the Viva Mulher, a cervical cancer control programme starting in 1997 as a pilot project in a cluster of states to become later nationalised in the following years. In assessing the main initiatives, actors, and the broader political context of the period, the article highlights the intricacies and the 
multifaceted nature of transforming regional initiatives into a comprehensive national programme.

A historical revision of these and other chapters in the fight against female cancers in South America, where breast and cervical cancer continue to occupy the first and second most frequent cancers amongst women between 15-44 years of age, may well broaden the appeal for a reconsideration of past health interventions that, it is hoped, would resonate beyond the discipline's work. 